\section{Endovascular Solution for Incorrect Stent Positioning}

A 58-year-old man presented to our service suffering from claudication with a walking distance of $100 \mathrm{~m}$. A preoperative angiography was performed showing an occlusion of the right common femoral artery with reperfusion of the superficial femoral artery 3 $\mathrm{cm}$ below the bifurcation, subtotal stenosis of the right common iliac artery with a residual lumen of $60-70 \%$, and subtotal stenosis of the right external iliac artery with a residual lumen of about $80 \%$ (Fig. 1). The vascular surgeons decided to perform a thromboendoarterectomy (TEA) of the right common femoral artery, but asked us to perform a preoperative percutaneous transluminal angioplasty and stenting of the right common iliac artery in order to create a sufficient run-in into the common femoral artery.

We decided to use a transaxillary approach with a vascular introducer of $7 \mathrm{Fr}$ and $45 \mathrm{~cm}$ and an angiographic guidewire (Terumo angled non-stiff 0.035 -inch, $180 \mathrm{~cm}$ in length) introduced into the iliac artery. Using the road-mapping technique primary stenting of the right common iliac artery with a self-expandable nitinol stent (Smart Cordis $10 \mathrm{~mm}$ memory $6 \mathrm{~cm}$ length) was performed. The first angiographic control after positioning, however, revealed malpositioning of the distal end of the stent into the internal iliac artery (Fig. 2). Access to the external iliac artery therefore became mandatory.

The guidewire (Terumo angled non-stiff 0.035 -inch) was easily passed through the meshes of the stent, although subsequent passage of angiographic catheters up to 4 Fr was difficult albeit ultimately successful. After expanding the pathway by the use of balloon catheters of 4 and $6 \mathrm{~mm}$ diameter (Wanda, Boston Scientific) another stent (Smart Cordis $10 \mathrm{~mm}$ memory $4 \mathrm{~cm}$ length) was placed with its proximal end in the common iliac artery and its distal end within the external iliac artery (Fig. 3). The stent was then expanded to $10 \mathrm{~mm}$ (balloon catheter $10 \mathrm{~mm}$ diameter $4 \mathrm{~cm}$ length; Wanda, Boston Scientific) at the upper end and up to $8 \mathrm{~mm}$ (balloon catheter $8 \mathrm{~mm}$ diameter and $4 \mathrm{~cm}$ length; Wanda, Boston Scientific) at the distal end after the iliac bifurcation.

The final angiographic control showed good results of the endovascular recanalization, with flow in the iliac axis down to the known occlusion and sufficient run-in for a subsequent femoral TEA (Fig. 4).

The patient underwent surgery $48 \mathrm{hr}$ later and is currently walking more than $200 \mathrm{~m}$ without pain (at 6 month follow-up).

Endovascular interventional radiology to treat the iliac axis is well known [1] and has been accepted for many years. However, experienced centers have also found that the techniques are not free from inadvertent complications [2], as in the case above, where our mistake was not to check the correct position of the guidewire by angiographic control but to trust fluoroscopy alone. Tricks and alternatives to solve even rare complications should be known in centers performing vascular interventions.

Stefano Barbero

Rita Di Savino

Carlo Tappero

Giovanni Gandini

Istituto Radiologia Universitaria Via Genova 3 10100, Turin, Italy e-mail: stefanobarbero@tele2.it

\section{References}

1. Reyes R, Carreira JM, Gude F, Gorriz E, Gallardo L, Pardo MD, Hermida M (2004). Long-term follow-up of iliac Wallstents. Cardiovasc Intervent Radiol 27:624-631. Epub Oct06, 2004. PMID: 15578139 [PubMed-in process] http://www.ncbi.nlm.nih.gov/entrez/query.fcgi? $\mathrm{cmd}=$ Retrieve $\& \mathrm{db}=$ pubmed $\&$ dopt $=$ Abstract $\&$ list_uids $=15578139$

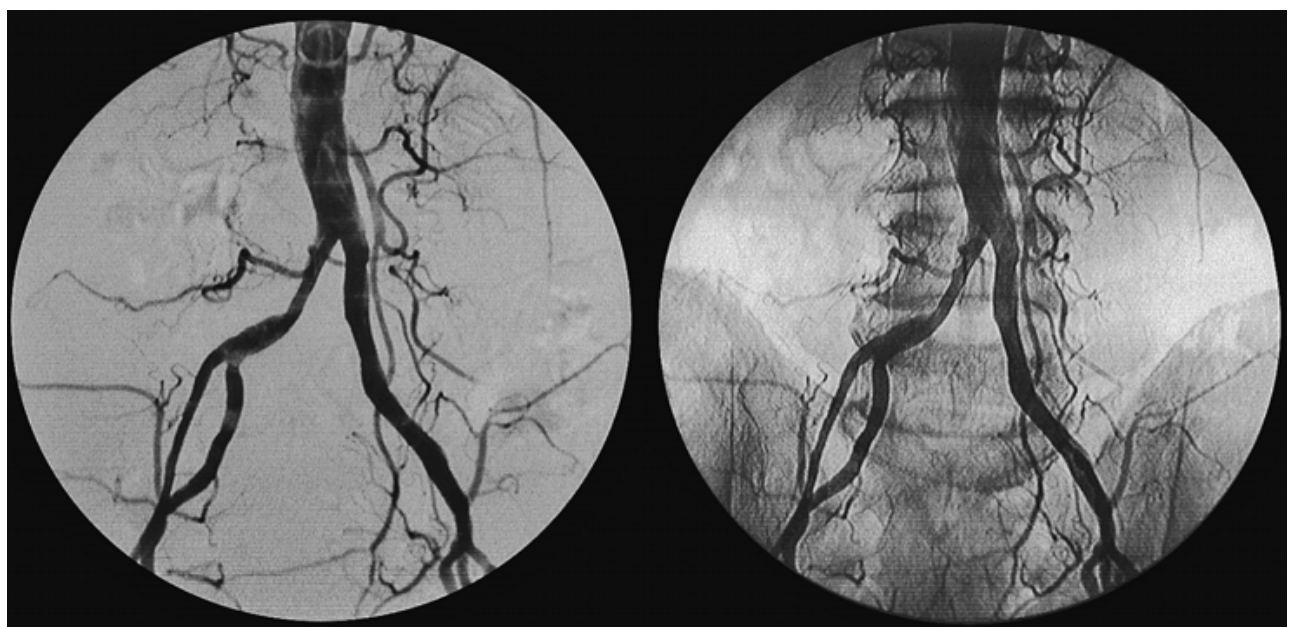

Fig. 1. Diagnostic angiographic of the iliofemoral tract: right common and external iliac artery subtotal stenoses. 


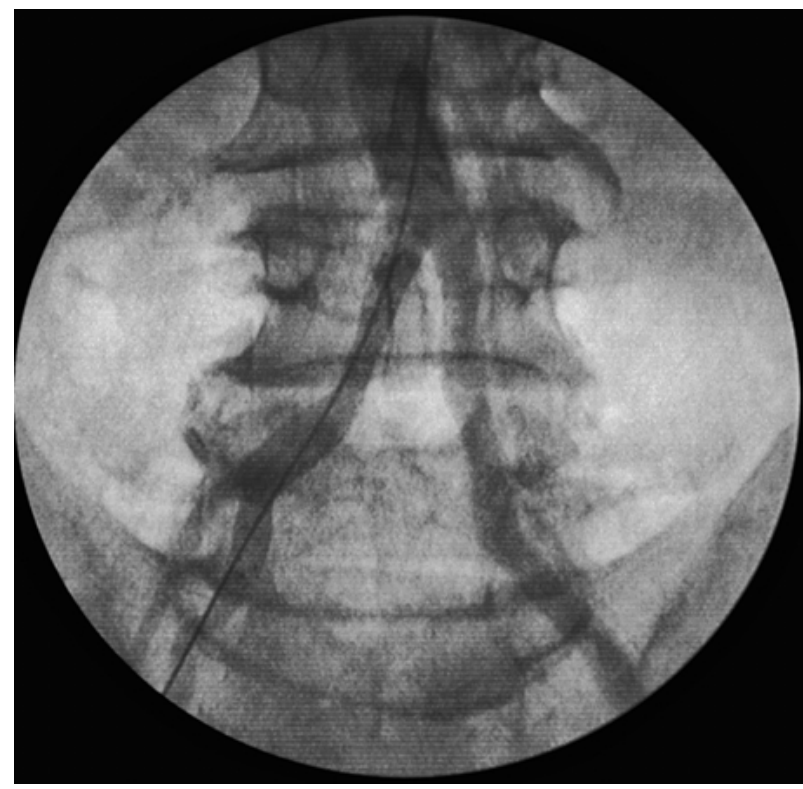

Fig. 2. Angiographic control after stenting: wrong distal positioning in the inner iliac artery.

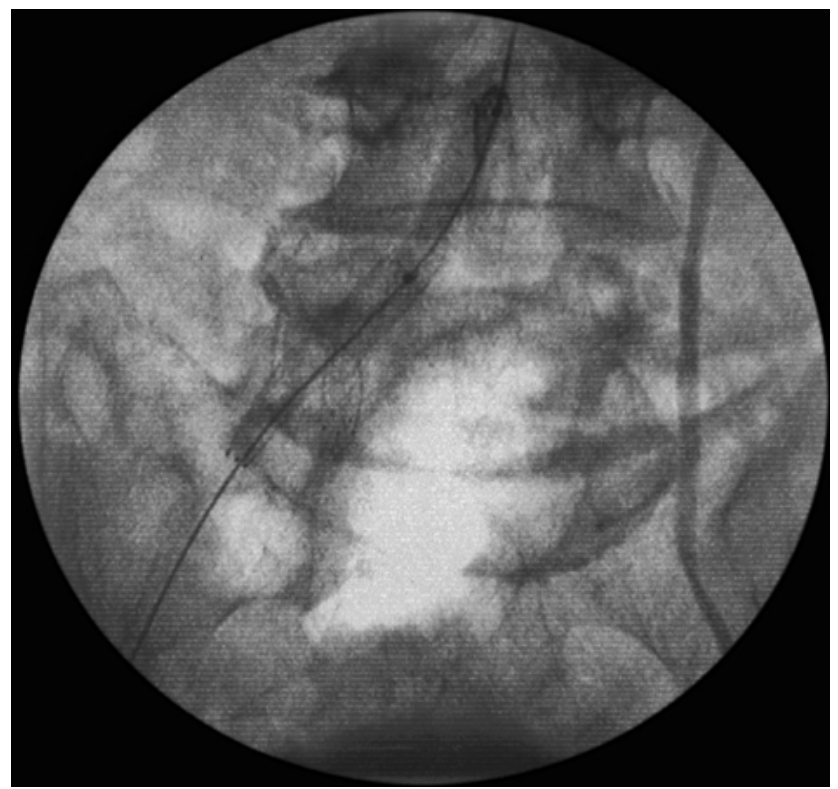

Fig. 3. Angiographic control after restenting.

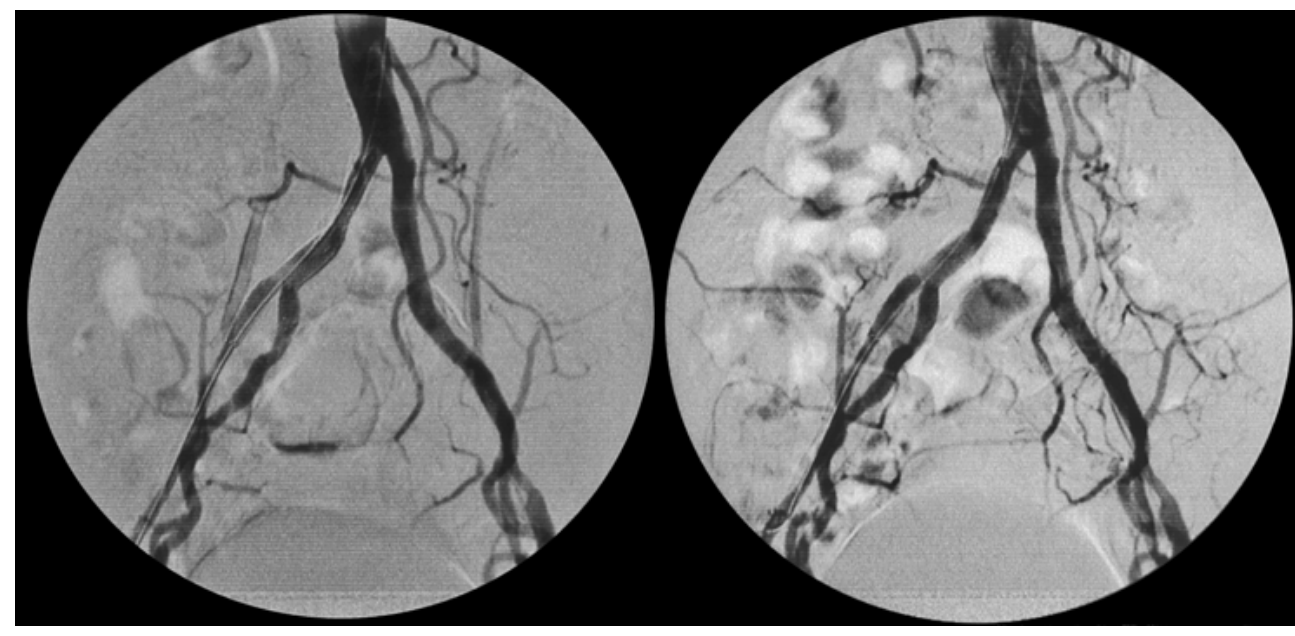

Fig. 4. Final angiographic control.

2. Parham WA, Puri S, Bitar SR, Kern MJ (2003) Management of iliac stent movement complicating peripheral vascular intervention: A rescue technique when stent deployment malfunctions. J Invas Cardiol
15:2772004 279. Epub May 2003: PMID: 12730638 [PubMed-indexed for MEDLINE] http://www.ncbi.nlm.nih.gov/entrez/query.fcgi?cmd= Retrieve \&db=pubmed\&dopt $=$ Abstract\&list_uids $=12730638$ 